\title{
Aspectos Morfocuantitativos de las Vellosidades Coriales Libres en Gestas Normales, con Diabetes, Hipertensión Arterial y Restricción del Crecimiento Intrauterino
}

\author{
Morphoquantitative Characteristics of Free Corial Vellosities in Normal Births, \\ with Diabetes, Hypertension and Restriction of Intrauterine Growth
}

Ruth Prieto Gómez ${ }^{1}$; Nicolás Ernesto Ottone ${ }^{2,8}$; Cristian Sandoval Vásquez ${ }^{3,4}$; Araceli Saavedra S. ${ }^{5}$ \& Homero Felipe Bianchi ${ }^{6,7}$

PRIETO, G. R.; OTTONE, N. E.; SANDOVAL, V. C.; SAAVEDRA, S. A. \& BIANCHI, H. F. Aspectos morfocuantitativos de las vellosidades coriales libres en gestas normales, con diabetes, hipertensión arterial y restricción del crecimiento intrauterino. Int. $J$. Morphol., 36(2):551-556, 2018.

RESUMEN: Las patologías de la gestación como la hipertensión, diabetes mellitus gestacional, o restricción del crecimiento intrauterino, pueden determinar modificaciones en las características morfológicas macro y microscópicas de la placenta y sus vellosidades coriales libres, y en el feto se puede acompañar de manifestaciones patológicas, con riesgo para su calidad de vida futura, e incluso su viabilidad. El objetivo de este trabajo consistió en describir aspectos morfométricos e histológicos de las vellosidades coriales libres en gestas normales, con diabetes e hipertensión arterial. Se utilizaron 30 placentas humanas y fueron separadas, según presencia o ausencia de patologías en el embarazo, en tres grupos: Normal (N), Síndrome Hipertensivo del Embarazo (SHE), Diabetes (D) y Restricción del Crecimiento Intrauterino (RCIU). Se usó ficha para registrar peso placentario y del recién nacido Todas las muestras fueron fijadas en formalina tamponada al $10 \%$. De cada una fueron extraídas 5 muestras, obteniendo 25 cortes por cada placenta. Posteriormente, fueron teñidas con H\&E, Azul Alcián y Tricrómico de Masson. Además, se efectuó el análisis histológico y morfométrico (Image ${ }^{\circledR}$ ) de las vellosidades coriales. El análisis estadístico fue realizado utilizando ANOVA. Entre los cambios morfológicos, se encontró una relación peso placentario/peso del recién nacido aumentada en la Diabetes Mellitus Gestacional asociada a cambios histológicos. No hubo cambios morfométricos significativos entre placentas N, SHE y D. Hubo un aumento en el número de vasos coriales en placentas del grupo $\mathrm{D}(\mathrm{P}<0,05)$ y de la superficie entre las vellosidades coriales. En el grupo SHE hubo aumento moderado de nudos sinciciales y presencia de fibrina en el estroma. Las placentas con Diabetes Mellitus Gestacional experimentan alteraciones histológicas, como consecuencia de cambios estructurales y funcionales. Además, el aumento de vasos sanguíneos en placentas con diabetes se produce por neoformación vascular y mayor penetración de vasos sanguíneos dentro de las vellosidades. En el caso del SHE las alteraciones placentarias se relacionan con la gravedad de la enfermedad.

PALABRAS CLAVE: Placenta; Vellosidades coriales libres; Diabetes mellitus gestacional; Hipertensión; Morfometría.

\section{INTRODUCCIÓN}

La estructura y la función de las vellosidades coriales libres de la placenta humana permiten al embrión y al feto un desarrollo normal. La existencia de patología durante la gestación, como la hipertensión arterial, la restricción del crecimiento y la diabetes gestacional producirán cambios macro y microscópicos en la morfología de la placenta y sus vellosidades, convirtiéndose en un factor de riesgo para el embrión y el feto.

\footnotetext{
${ }^{1}$ Departamento de Pediatría y Cirugía Infantil, Facultad de Medicina, Universidad de La Frontera, Temuco, Chile.

${ }^{2}$ Laboratorio de Plastinación \& Técnicas Anatómicas, Centro de Investigación en Ciencias Odontológicas (CICO), Facultad de Odontología, Universidad de La Frontera, Temuco, Chile.

${ }^{3}$ Programa de Doctorado en Ciencias Morfológicas, Facultad de Odontología, Universidad de La Frontera, Temuco, Chile.

${ }^{4}$ CIMA - Centro de Investigación en Morfología Aplicada, Reconstructiva y Forense, Universidad de La Frontera, Temuco, Chile.

${ }^{5}$ Departamento de Pediatría y Cirugía Infantil, Facultad de Medicina, Universidad de La Frontera, Temuco, Chile.

${ }^{6}$ Departamento de Anatomía, Facultad de Medicina, Universidad de Buenos Aires, Buenos Aires, Argentina.

${ }^{7}$ IUCS Fundación Barceló, Departamento de Anatomía, Buenos Aires, Argentina.

${ }^{8}$ Centro de Excelencia en Estudios Morfológicos y Quirúrgicos (CEMyQ), Universidad de La Frontera, Temuco, Chile.

Financiado por Universidad de La Frontera, Proyecto DIUFRO Nº DI16-0074
} 
PRIETO, G. R.; OTTONE, N. E.; SANDOVAL, V. C.; SAAVEDRA, S. A. \& BIANCHI, H. F. Aspectos morfocuantitativos de las vellosidades coriales libres en gestas normales, con diabetes, hipertensión arterial y restricción del crecimiento intrauterino. Int. J. Morphol., 36(2):551-556, 2018.

En la Diabetes Mellitus Gestacional (DMG) existe una alteración en el crecimiento fetal, que podría estar relacionada con un déficit en la oxigenación del feto y cambios en el transporte transplacentario de los nutrientes, aumentando la disponibilidad de muchos de ellos al feto y ocasionando el sobrecrecimiento fetal, y en relación al peso placentario/peso fetal está aumentado en relación a la gestación normal y el aumento en el peso placentario se ha asociado principalmente a cambios a nivel histológico, principalmente a fenómenos de hiperproliferación e hipervascularización (Castejón \& Molinaro, 2004; Roa et al., 2012).

En el Síndrome Hipertensivo del Embarazo (SHE), las vellosidades coriales, en las gestantes con desordenes hipertensivos, sufren un proceso de acelerada maduración; un envejecimiento rápido puede dar origen a una temprana apoptosis o necrosis del trofoblasto que provocaría una separación de las vellosidades de anclaje, fijas en la placa basal, en consecuencia el desprendimiento de la placenta sería inminente. Pero también durante la gestación normal pueden provocarse disturbios en el proceso de maduración de las vellosidades coriales, que pueden acelerar o retardar su crecimiento (Maly et al., 2005).

La Restricción del Crecimiento Intrauterino (RCI) está asociado a un aumento en la morbilidad y la mortalidad perinatal, siendo una de las principales complicaciones de la gestación y efectos que pueden perdurar hasta la adultez (Beauharnais et al., 2012; Tennant et al., 2014) como riesgo de patologías cardiovasculares y la diabetes. Es necesario contar con un conocimiento de la morfología placentaria normal para poder interpretar los cambios producidos por la RCI (Bernirschke \& Kaufmann, 2000).

De acuerdo a la evidencia presentada el objetivo de este estudio consistió en realizar una descripción de las características morfológicas, macro y microscópicas, de la placenta humana y sus vellosidades coriales libres en gestas sin patología y gestas con hipertensión, diabetes y restricción del crecimiento intrauterino.

\section{MATERIAL Y MÉTODO}

Diseño de Investigación. Estudio de corte transversal del tipo descriptivo-inferencial, y por conveniencia. Se utilizaron 30 placentas, de mujeres entre 17 y 43 años de edad, durante los meses de julio 2016 a marzo de 2017 de la maternidad del Hospital Hernán Henríquez Aravena, de Temuco. Los criterios de inclusión considerados fueron: Parto de término, embarazo único, recién nacido vivo, hipertensión (SHE), diabetes. Los criterios de exclusión fueron: embarazo ectópico, embarazo molar y desprendimiento prematuro de placenta normoinserta. El protocolo de investigación que se desarrolló en esta investigación contó con la aprobación del Comité Ético Científico del Servicio Salud Araucanía Sur de Temuco, de Resolución exenta $N^{\circ} 1179$, del 6 de marzo de 2014. A todas las madres se les solicitó consentimiento informado, aprobado por el mismo Comité de Ética, para la utilización de la placenta en el estudio.

Recolección de los Datos. La recolección de los datos implicó:

Instrumento de medición. Para los aspectos macroscópicos se utilizó una balanza calibrada para el peso de las placentas, además huincha y regla de uso exclusivo para el estudio, registrado en ficha de registro y posterior tabulación.

Métodos para la recolección de los datos. Para la identificación de la muestra se elaboró una ficha asignando un código a cada placenta para mantener la confidencialidad, donde se registró: edad de la madre, gestación, paridad, tipo de parto, sexo del RN, peso del RN, diagnóstico pediátrico y patología de la madre según protocolo del estudio. De la placenta y como protocolo se registró peso, grosor, diámetro, número de cotiledones, forma, sitio inserción del cordón, presencia o ausencia de calcificaciones y color de la placenta.

Analisis Histológico de las Muestras. Las placentas fueron fijadas en formalina tamponada al $10 \%$. Se extrajeron cortes de la porción central de la placenta. De cada muestra placentaria, se obtuvieron cinco placas, y en total 25 placas por placenta. Se utilizaron técnicas histológicas como H-E, azul de Alcian, y Tricrómico de Masson. Para el análisis histológico se utilizó Microscopio NIKON y una cámara digital incluida NIKON E 4.500. (Figs. 1, 2, 3).

Para el análisis de las placentas se constituyeron los siguientes grupos: Grupo control $(\mathrm{N})$ placentas de gestas sin patología, Grupo (SHE) placentas de gestas con SHE y Grupo (RCIU) placentas con restricción del crecimiento intrauterino, Grupo (D): placentas de gestas con diabetes y Grupo (O) placentas con SHE y RCUI.

En cada grupo, se analizarán los parámetros macroscópicos de las placentas, en relación a: a) Peso, b) longitud, c) diámetro mayor y menor d) espesor e) presencia de calcificaciones y f) número de cotiledones.

Con respecto al análisis microscópico/histológico, se seleccionaron las vellosidades libres cortadas en forma transversal y se consideraron los siguientes parámetros para ser evaluados: a) área de la vellosidad; b) perímetro de la vellosidad; c) Número de vasos sanguíneos por vellosidad; d) número de zonas alfa; e) zonas beta por vellosidad. 
PRIETO, G. R.; OTTONE, N. E.; SANDOVAL, V. C.; SAAVEDRA, S. A. \& BIANCHI, H. F. Aspectos morfocuantitativos de las vellosidades coriales libres en gestas normales, con diabetes, hipertensión arterial y restricción del crecimiento intrauterino. Int. J. Morphol., 36(2):551-556, 2018.

Para la morfometría se utilizó el programa computacional Image $®$

Analisis estadístico de los datos. Para el análisis cuantitativo se usó estadística descriptiva e inferencial, para describir y realizar análisis estadístico.

Los datos fueron analizados mediante estadística descriptiva (Media DE) se realizó adicionalmente pruebas de normalidad de las mediciones de los datos a través de la prueba de Kolgomorov-Smirnov, además, se estableció la relación de las mediciones entre el grupo de control y los grupos de casos a través de un ANOVA de una vía, en caso de diferencias significativas entre los grupos se realizó la prueba de Scheffé. Los datos fueron analiza-

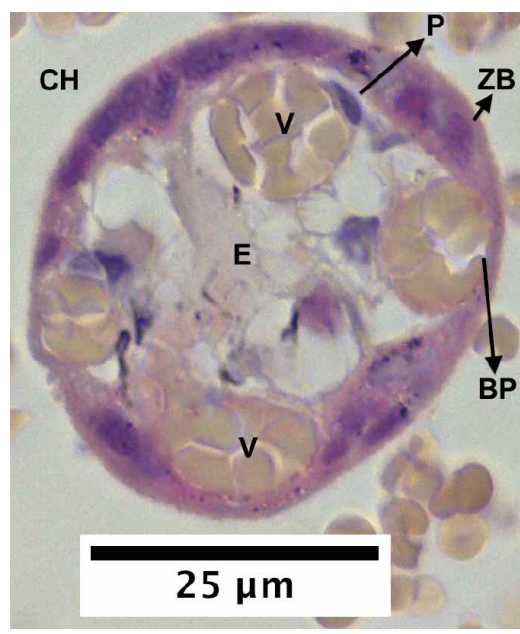

Fig. 1. Vellosidad corial libre correspondiente al grupo control (N). (V) Vasos sanguíneos, (E) Estroma de la vellosidad, (ZB) Zona Beta, (BP) Barrera Placentaria, (CH) Cámara hemática, (P) Pericito.

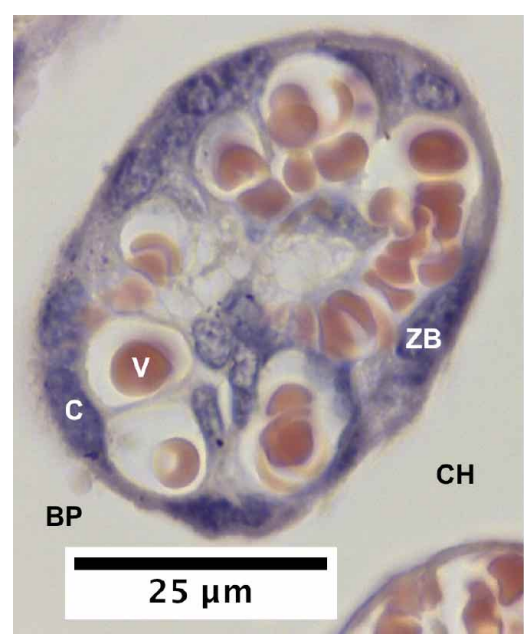

Fig. 2. Vellosidad corial libre correspondiente al grupo (D). La barrera placentaria tiene un mayor grosor que en el grupo $\mathrm{N}$. (V) Vasos sanguíneos, (CT) Citotrofoblasto, (ZB) Zona Beta, (BP) Barrera Placentaria, (CH) Cámara hemática. dos con el software SPSS/PC + versión 20.0, SPSS, Chicago, IL, un valor de $\mathrm{p}<0,05$ siendo elegido como umbral para significancia.

\section{RESULTADOS}

El área de las placentas del grupo control y los grupos estudio SHE y Diabetes (D) no presentaron diferencias significativas con los valores encontrados. El grupo otras patologías $(\mathrm{O})$ sí presentó diferencias significativas con el grupo de SHE y con de RCI, igual resultado mostró la medición del perímetro de las vellosidades. Todos los resultados pueden encontrarse en la Tabla I.

Tabla I. Parámetros morfológicos analizados en los diferentes grupos. a. Diferencias significativas $(\mathrm{P}<0,05)$ con el grupo SHE. b. Diferencias significativas $(\mathrm{P}<0,05)$ con el grupo RCU.

\begin{tabular}{|c|c|c|c|c|c|c|}
\hline & $\mathrm{N}$ & SHE & $\mathrm{RCU}$ & $\mathrm{D}$ & $\mathrm{O}$ & \\
\hline \multirow[t]{2}{*}{ Área } & 1282,528 & 1770,882 & 1941,505 & 1101,819 & $1005,305^{\mathrm{ab}}$ & \multirow[b]{2}{*}{0,002} \\
\hline & 560,124 & 1029,352 & 1493,490 & 463,716 & 245,560 & \\
\hline \multirow[t]{2}{*}{ P erímetro } & 129,604 & 149,858 & 154,933 & 119,883 & $116,707 \mathrm{a}^{\mathrm{b}}$ & \multirow[b]{2}{*}{0,002} \\
\hline & 27,902 & 43,478 & 55,067 & 28,655 & 14,632 & \\
\hline \multirow[t]{2}{*}{ Diámetro mayor } & 41,905 & 45,849 & 51,489 & 39,438 & $38,143^{b}$ & \multirow{2}{*}{0,028} \\
\hline & 9,294 & 15,988 & 18,107 & 10,949 & 5,875 & \\
\hline \multirow[t]{2}{*}{ Diámetro menor } & 35,729 & 38,873 & 41,333 & 31,770 & 30,690 & \multirow{2}{*}{0,023} \\
\hline & 8,715 & 14,450 & 15,947 & 7,638 & 4,567 & \\
\hline \multirow[t]{2}{*}{$\mathrm{N}^{\mathrm{o}}$ vasos sanguíneos } & 3,760 & 4,040 & 4,200 & 4,000 & 2,880 & \multirow{2}{*}{0,179} \\
\hline & 1,422 & 2,653 & 1,814 & 2,261 & 1,130 & \\
\hline \multirow[t]{2}{*}{ Zona Alfa } & 3,280 & 3,600 & 3,100 & 3,500 & 3,200 & \multirow{2}{*}{0,616} \\
\hline & 0,936 & 1,354 & 0,316 & 1,179 & 1,041 & \\
\hline \multirow[t]{2}{*}{ Zona Beta } & 3,640 & 4,360 & 3,700 & 4,000 & $3,160 \mathrm{~b}$ & \multirow{2}{*}{0,033} \\
\hline & 1,114 & 1,868 & 0,949 & 1,247 & 0,898 & \\
\hline
\end{tabular}


PRIETO, G. R.; OTTONE, N. E.; SANDOVAL, V. C.; SAAVEDRA, S. A. \& BIANCHI, H. F. Aspectos morfocuantitativos de las vellosidades coriales libres en gestas normales, con diabetes, hipertensión arterial y restricción del crecimiento intrauterino. Int. J. Morphol., 36(2):551-556, 2018.

El diámetro mayor de las vellosidades coriales libre no mostró diferencias significativas entre los grupos, con excepción del diámetro promedio del grupo $\mathrm{O}$ con respecto al grupo SHE. En el caso del diámetro menor no hubo diferencias significativas entre los grupos.

En el número de vasos, a pesar de no presentar diferencias significativas, el grupo de RCI tiene un promedio de 4,2 vasos por vellosidad, el mayor de entre los grupos.

En relación a la presencia de zonas alfa, el grupo de SHE presenta mayor número de ellas, no siendo significativa la diferencia entre los grupos.

Las zonas beta presentan el promedio más alto en el grupo de RCI. El grupo $\mathrm{O}$ muestra una diferencia significativa con el grupo de RCU.

De las madres del estudio. El promedio de edad de las madres fue de 25 años, siendo el máximo de 43 años y el mínimo de 17 años. El promedio de la talla materna fue de 1,58 $\mathrm{cm}$, la mínima talla $1,42 \mathrm{~m}$ y la máxima de $1,70 \mathrm{~m}$. En relación al peso materno el $32 \%$ correspondía a peso normal, el $40 \%$ sobrepeso y el $27,5 \%$ era obesa. El tipo de parto fue en su mayoría parto normal con un $65 \%$ y el parto cesáreo el $35 \%$

De los recién nacidos. El peso de los recién nacidos 3.457 g, $4.810 \mathrm{~g}$ el peso máximo y $2.340 \mathrm{~g}$ el menor. La edad gestacional promedio fue de 38 semanas, 33 semanas de gestación la mínima y 41 semana la máxima. La mayoría de los recién nacido fue de sexo femenino, $55 \%$ de del total.

De las placentas del estudio. El peso placentario fue en promedio de 569,37 g, la mínima de 360 g y la máxima de $877 \mathrm{~g}$. La longitud del cordón umbilical fue en promedio de $67,87 \mathrm{~cm}$, el mínimo de $45 \mathrm{~cm}$ y el máximo de $90 \mathrm{~cm}$. El diámetro promedio fue de $20,1 \mathrm{~cm}$, el promedio mínimo fue de $12 \mathrm{~cm}$ y el máximo de $30 \mathrm{~cm}$. Los cotiledones fueron en promedio 16, el mínimo 8 y el máximo 25 . El color de las placentas fue en su mayoría rojo oscuro, $90 \%$, rosado un 2,5 $\%$, verde o impregnada de meconio con $5 \%$, y otros $2,5 \%$. Un $77 \%$ de las placentas tenía presencia de calcificaciones.

\section{DISCUSIÓN}

En las placentas con diabetes gestacional, la distancia entre la circulación materna y la fetal está aumentada debido a un aumento de la superficie entre las vellosidades coriales, así como al mayor grosor de la membrana basal del sincitiotrofoblasto por un depósito aumentado de colageno tipo IV a este nivel. El estroma entre las vellosidades se encuentra edematoso y hay un aumento de las células de Hofbauer (macrófagos placentarios encargados de la sintesis de leptina, TNF-a e interleukinas), lo que va a modificar la función metabólica y endocrina de estas placentas. También se describe un aumento de la superficie capilar por fenómenos de neoformación vascular y una mayor penetración de estos vasos dentro de las vellosidades. Este fenómeno de hipervascularizacion es provocado por factores proangiogénicos para compensar la alteración en la difusión materno-fetal de algunos nutrientes, especialmente de oxígeno (Castejón \& Molinaro). Una manera de explicar el aumento placentario está en relación con la lámina basal de los capilares coriónicos, que es parte de la barrera placentaria, por lo que el aumento de su espesor incrementara el grosor de la barrera placentaria, lo cual puede conducir a una reducción del transporte de oxígeno y otros nutrientes a través de la barrera y en respuesta a esta disminución de la pO2, las vellosidades terminales muestran una hiperplasia parcialmente responsable del aumento en el peso de la placenta (Ashfaq et al., 2005; Aires \& Dos Santos, 2015).

En relación con el sindrome hipertensivo, las vellosidades intermedias inmaduras, en una placenta madura normal, se localizan en la porción central del placentoma o lóbulo placentario, son vellosidades que todavía proliferan y representan una clase de reserva del crecimiento (Baumüller et al., 2015). Maly et al. determinaron que los exámenes macro y microscópicos de las placentas de embarazos con preeclampsia mostraron cambios isquémicos tales como formación de nudos sinciales prominentes y áreas de marcada ramificación antigénica. Se encontró necrosis fibrinoide y depósito intramural de lípidos en las paredes de los vasos uterinos (aterosis aguda) en gran parte de las placentas, y el examen macroscópico reveló la presencia de infartos en 6 placentas, que afectó a menos del $10 \%$ del volumen de la placenta en cada caso. En caso de hipertensión materna, Ashfaq et al. establecieron que la aterosclerosis afecta a los vasos sanguíneos del útero, estrechando su luz, lo que conduce a una reducción del flujo sanguíneo en el espacio intervelloso. En relación a la placenta, hay mayor desarrollo de infartos, coágulos de sangre, hematomas, aterosis, y un menor peso y volumen de la placenta (Karlsson et al., 1982).

Prins et al. (2012) establecen en su investigación, la participación del sistema inmunológico materno en el remodelado de la arteria espiral uterina, la invasión del trofoblasto y la aceptación simultánea del feto semialogénico durante el embarazo. Ante una desregulación del sistema inmune materno, producto de la hipertensión 
materna, en deciduas del primer trimestre, se produce una mayor expresión de IL6 y de ARNm de macrófagos, encontrándose menores proporciones de macrófagos reguladores. A su vez detectaron una menor expresión de Gata3 (Th2) ARNm en deciduas de los embarazos con posterior restricción del crecimiento fetal. De esta manera confirman la aparición de resultados adversos en placentas del tercer trimestre asociadas a trastornos inmunológicos producto de la hipertensión del embarazo.

Almasry et al. (2012) identificaron que el número medio de arterias vellositarias del tallo y el número medio de capilares vellosos terminales por campo son significativamente menores en el grupo con restricción del crecimiento intrauterino idiopático que en el grupo control. Además encontraron una correlación significativa entre el peso al nacer y las diferentes características patológicas en las vellosidades del tallo como el número, estrechamiento arterial, cambios degenerativos y villitis. También identificaron una correlación significativa entre el peso neonatal al nacer y el número de capilar velloso terminal pero no encontraron una correlación significativa entre el peso al nacer y los cambios fibróticos vellosos terminales.

\section{CONCLUSIÓN}

Las vellosidades terminales, de placentas con diabetes gestacional, muestran una hiperplasia parcialmente responsable del aumento del peso de la placenta.

La hipertensión arterial en la gestación, relaciona la placenta con un mayor desarrollo de infartos, presencia de zonas beta y un menor peso de la placenta.

Las zonas beta presentaron el mayor promedio en el grupo de restricción del crecimiento, y se identificó una correlación significativa entre el peso del recién nacido y peso de la placenta y el número de vasos de las vellosidades terminales libres de la placenta.

PRIETO, G. R.; OTTONE, N. E.; SANDOVAL, V. C.; SAAVEDRA, S. A. \& BIANCHI, H. F. Morphoquantitave characteristics of free corial vellosities in normal births, with diabetes, hypertension and restriction of intrauterine growth. Int. J. Morphol., 36(2):551-556, 2018.

SUMMARY: Gestational pathologies such as hypertension, gestational diabetes mellitus and restriction of intrauterine growth can determine changes in the macro and microscopic morphological characteristics of the placenta and its free chorionic villi. In the fetus it can be accompanied by pathological manifestations with risk to its viability and future quality of life. The aim of this work was to describe morphometric and histological aspects of free chorionic villi in normal pregnancies associated with diabetes, hypertension and restriction of intrauterine growth. Thirty human placentas were used and were separated into three groups: Normal (N), Hypertensive Pregnancy Syndrome (SHE), Diabetes (D), and Restriction of Intrauterine Growth (RIG) according to evident pathologies or absence thereof during pregnancy. Tab was used to record placental and newborn weight. All samples were fixed in $10 \%$ buffered formalin. From each, 5 samples were extracted, obtaining 25 cuts for each placenta. Subsequently, they were stained with $\mathrm{H} \& \mathrm{E}$, Alcian Blue and Masson's Trichrome. In addition, histological and morphometric analysis (Image $®$ ) of the chorion villus was carried out. Statistical analysis was performed using ANOVA. Among the morphological changes, an increased placental weight / weight ratio of the newborn was found in Gestational Diabetes Mellitus associated with histological changes. There were no significant morphometric changes between placentas N, SHE and D. There was an increase in the number of corial vessels in placentas of group $\mathrm{D}(\mathrm{P}<0.05)$ and of the surface between the chorion villi. In the SHE group there was a moderate increase in syncytial nodes and presence of fibrin in the stroma. Placentas with Gestational Diabetes Mellitus experience histological alterations, as a consequence of structural and functional changes. In addition, the increase of blood vessels in placentas $\mathrm{D}$ is produced by vascular neoformation and increased penetration of blood vessels into the villi. In the case of SHE, placental alterations are related to the severity of the disease.

KEY WORDS: Placenta; Free chorionic villi; Gestational diabetes mellitus; Hypertension; Morphometry.

\section{REFERENCIAS BIBLIOGRÁFICAS}

Aires, M. B. \& Dos Santos, A. C. Effects of maternal diabetes on trophoblast cells. World J. Diabetes, 6(2):338-44, 2015.

Almasry, S. M.; Eldomiaty, M. A.; Elfayomy, A. K.; Habib, F. A. \& Safwat, M. D. Structural analysis of human placental stem and terminal villi from normal and idiopathic growth restricted pregnancies. J. Mol. Histol., 43(3):263-71, 2012.

Ashfaq, M.; Janjua, M. Z. \& Channa, M. A. Effect of gestational diabetes and maternal hypertension on gross morphology of placenta. J. Ayub. Med. Coll. Abbottabad., 17(1):44-7, 2005.

Baumüller, S.; Lehnen, H.; Schmitz, J.; Fimmers, R. \& Müller, A. M. The impact of insulin treatment on the expression of vascular endothelial cadherin and Beta-catenin in human fetoplacental vessels. Pediatr. Dev. Pathol., 18(1):17-23, 2015.

Beauharnais, C. C.; Roberts, D. J. \& Wexler, D. J. High rate of placental infarcts in type 2 compared with type 1 diabetes. J. Clin. Endocrinol. Metab., 97(7):E1160-4, 2012.

Bernirschke, K. \& Kaufmann, P. Pathology of the Human Placenta. $4^{\text {th }}$ ed. New York, Springer-Verlag, 2000.

Castejón, O. C. \& Molinaro, M. P. V. Maturity of the chorionic villi and its relation with hypertensive disorders in cases of severe premature placental detachment of normal insert placenta. Rev. Fac. Cienc. Salud Univ. Carabobo, 8(3):17-26, 2004 
PRIETO, G. R.; OTTONE, N. E.; SANDOVAL, V. C.; SAAVEDRA, S. A. \& BIANCHI, H. F. Aspectos morfocuantitativos de las vellosidades coriales libres en gestas normales, con diabetes, hipertensión arterial y restricción del crecimiento intrauterino. Int. J. Morphol., 36(2):551-556, 2018.

Karlsson, K.; Ljungblad, U. \& Lundgren, Y. Blood flow of the reproductive system in renal hypertensive rats during pregnancy. Am. J. Obstet. Gynecol., 142(8):1039-44, 1982.

Maly, A.; Goshen, G.; Sela, J.; Pinelis, A.; Stark, M. \& Maly, B. Histomorphometric study of placental villi vascular volume in toxemia and diabetes. Hum. Pathol., 36(10):1074-9, 2005.

Prins, J. R.; Faas, M. M.; Melgert, B. N.; Huitema, S.; Timmer, A.; Hylkema, M. N. \& Erwich, J. J. Altered expression of immune-associated genes in first-trimester human decidua of pregnancies later complicated with hypertension or foetal growth restriction. Placenta, 33(5):453-5, 2012.

Roa, I.; Smok, S. C. \& Prieto, G. R. Placenta: Compared anatomy and histology. Int. J. Morphol., 30(4):1490-6, 2012.

Tennant, P. W.; Glinianaia, S. V.; Bilous, R. W.; Rankin, J. \& Bell, R. Preexisting diabetes, maternal glycated haemoglobin, and the risks of fetal and infant death: a population-based study. Diabetologia, 57(2):28594, 2014.

\author{
Dirección para correspondencia \\ Ruth Prieto Gómez \\ Departamento de Pediatría y Cirugía Infantil \\ Facultad de Medicina \\ Universidad de La Frontera \\ Temuco \\ CHILE
}

E-mail: ruth.prieto@ufrontera.cl

Recibido : 21-12-2017

Aceptado: 07-02-2018 